\title{
The Yellow Vests Movement - a case of long transient dynamics?
}

\author{
Andrew Morozov ${ }^{a}$, Sergei Petrovskii ${ }^{a 1}$ and Sergey Gavrilets ${ }^{b}$ \\ ${ }^{a}$ Department of Mathematics, University of Leicester, Leicester, LE1 7RH, UK \\ ${ }^{b}$ Department of Ecology \& Evolutionary Biology, Department of Mathematics, National \\ Institute for Mathematical and Biological Synthesis (NIMBioS), Center for the Dynamics of \\ Social Complexity (DySoC), University of Tennessee, Knoxville, TN 37996, USA
}

May 29, 2019

\begin{abstract}
Understanding the dynamics of protests and social unrest is important in order to ensure a stable, sustainable development of the society. Mathematical models of social dynamics have been increasingly recognized as a powerful research tool in achieving this goal. Here, motivated by the fact that the dynamics of the ongoing Yellow Vest Movement in France exhibit anomalously long duration (currently it is in 30th week), we explore whether this can be a result of a dynamical systems phenomenon known as long transients. To this end, we build and study a hierarchy of mathematical models describing the "population dynamics" of the movement, i.e. how the number of protesters changes with time. We show that in these models long transients appear via two roots: via a ghost attractor and via an interaction of the slow and fast dynamics. We demonstrate that long transients are also present in some earlier models of social protests. Interestingly, our models predict that the Yellow Vest Movement should end abruptly by, at the latest, mid-summer 2019 without any interference from the French government. More generally, we argue that long transients are a generic feature of dynamical models describing social processes in the same way as they are in models of physical, ecological, and evolutionary systems.
\end{abstract}

Keywords: social dynamics; yellow vests; regime shift; long transients; ghost attractor

\footnotetext{
${ }^{1}$ Corresponding author. Email: sp237@le.ac.uk
} 


\section{Introduction}

In September of 2018, the French Government presented its budget for 2019. One of the announced measures was an increase in fuel taxes on January 1, 2019: 6.5 cents per liter on diesel and 2.9 cents per litre on petrol. A stated reason for these increases was to fight global warming via reduced fuel consumption. The taxes on petrol and diesel in France were already increasing throughout 2018 causing widespread discontent. The Government's plans for 2019 were seen as the final straw. At the end of October, two 30-year-old truck drivers create a Facebook event to "block all French roads" on November 17th to protest the tax hikes. Related posts and videos on Facebook attracted millions of views. One of those videos suggested the idea of wearing yellow vests (which each French driver is required by law to have in their vehicle) during the protests. On Saturday November 17, about 290,000 people joined mass demonstrations across France and the Yellow Vests Movement was born. The movement is populist and grass-root. Many of the demonstrations happening since then turned violent leading to 15 deaths, about four thousand of injuries, and almost nine thousand of arrests.

France, as many other countries in the world, has seen its share of social unrest, e.g. violent riots in 2005 (Bonnasse-Gahot et al., 2018) and the mass anti-gay-marriage protests in January 2013 (BBC News, 2013). Most of them quickly subside in a few days or a couple of weeks. This is what seemed to be happening in France in December of 2018 as the Christmas and the New Year holidays were approaching. However the street actions, which usually take place on Saturdays, resumed with a new energy in mid-January. The Yellow Vests Movement appears now to be quite unique in its duration: it is currently in its 30 th week.

An important and interesting question is why the Yellow Vests Movement is so persistent. Naturally, there are different perspectives on the duration of social unrest including psychological, sociological, political and economic ones offering various explanations (Bonnasse-Gahot et al., 2018, Davies et al., 2012, Pires and Crooks, 2017, Schussman and Soule, 2005). Here we approach this question from the point of view of complex dynamical systems. Mass manifestations and riots specifically, and collective behaviors more generally, are examples of complex social processes which can be described by dynamic systems (Andreev et al., 1997, Berestycki et al., 2015, Bonnasse-Gahot et al., 2018, Khosaeva, 2015) and as such are expected to exhibit certain generic dynamics. In particular, there is a striking similarity between the slow decay in participation in the Yellow Vests Movement and the phenomenon of long transient dynamics in physical and biological systems (Hastings and Higgins, 1994, Hastings et al., 2018, Lai and Tél, 2011, Van Geest et al., 2005).

In a nutshell, a long transient means that the dynamical system appears to be approaching its stable asymptotical dynamics (e.g. steady-state, periodical or chaotic) while in fact these dynamics are only transient. The quasi-asymptotical dynamics can persist for a long time before the system experiences a fast transition to its true asymptotics, sometimes distinctly different from the transient regime. In a non-spatial system, the main mechanisms resulting in long transient are ghost attractors (e.g. as arising from a saddle-node bifurcation: for parameter values just past their bifurcation value, the system mimics its steady state dynamics prior to the bifurcation), crawl-bys (slow changes near saddle points) and slow-fast dynamics 
(different processes take place on different time scale); for more details, see (Hastings et al., 2018). In spatial systems, there are additional mechanisms that include travelling waves and formation of transient spatiotemporal patterns (Lai and Tél, 2011, Morozov et al., 2019, Petrovskii et al., 2005).

The existence of long transients has been demonstrated in physical (Lai and Tél, 2011), ecological (Hastings and Higgins, 1994, Hastings et al., 2018, Morozov et al., 2019), evolutionary (Gavrilets and Hastings, 1995, 1998), and economics (Jakimowicz, 2010, 2013) systems. However, to our knowledge the possibility of long transients has never been explicitly discussed within the context of social behaviors.

Achieving a better understanding of the dynamics of protests and riots as well as social unrest more generally by using mathematical modelling has gained a growing recognition recently as a powerful research approach (Andreev et al., 1997, Berestycki et al., 2015, Berestycki and Rodriguez, 2016, Bonnasse-Gahot et al., 2018, Brantingham and et al., 2012, Davies et al., 2012, Eguiluz et al., 2006, Epstein, 1997, 2002, Fonoberova and et al., 2012, Khosaeva, 2015, Smith et al., 2012, Turalska et al., 2013, Turchin, 2003, Turchin et al., 2018). Indeed, since replicated sociological experiments with large groups of people are rarely possible (and it is hardly possible at all to simulate social unrest under controlled conditions), capturing the complexity of the social dynamics through tractable experiments is not feasible. Mathematical modelling and computer simulations create a virtual environment where hypotheses can be tested and different scenarios investigated in detail, and hence provide a feasible alternative to the experiment. A variety of approaches and techniques have been developed and used such as threshold models (Centola, 2013, Granovetter, 1978, Macy, 1991), evolutionary game theory models (Davies et al., 2012, Gavrilets, 2015, Whitehouse et al., 2017), social network models (Eguiluz et al., 2006, Turalska et al., 2013), agent-based models (Epstein, 2002, Fonoberova and et al., 2012) and epidemiological models (Bonnasse-Gahot et al., 2018, Epstein, 1997, Khosaeva, 2015), both in spatial and nonspatial systems. The models range from relatively simple, allowing only for some basic feature of the phenomenon (Epstein, 2002), to more detailed ones that endeavour to take into account the full complexity of the social dynamics (Davies et al., 2012, Gavrilets, 2015). The case studies include a few well-known events such as the London riots of 2011 and the French riots 2005, in particular aiming to describe the intensity and timing of the unrest and to analyze the processes and mechanisms involved (Bonnasse-Gahot et al., 2018, Davies et al., 2012).

Our goal here is to offer simple models of these processes to illustrate potential importance of long transients. We will build a family of models of increased complexity that describe the slow decay in the number of the Yellow Vest protesters and explain the dynamical mechanisms resulting in the slow decay. In doing this, we show that the slow decay is endemic, either being caused by a ghost attractor that arises following the regime shift (bifurcation) or occurring as a slow phase of the slow-fast dynamics. Based on the properties of the models, we predict that the end of the protests will happen abruptly and provide a range of expected dates for that. We also discuss the difference in the dynamical properties that makes the Yellow Vest Movement different from other recent events of social unrest. 


\section{Mathematical models}

In this section, we develop several mathematical models for the dynamics of protests and apply them to the data on the Yellow Vest Movement. We introduce the models in the order of increasing complexity. For all models, we describe the dynamics of protests in terms of average, 'mean-field' values, hence disregarding any explicit effects of stochasticity: arguably, this approach works well when applied to sufficiently large systems ${ }^{2}$, i.e. sufficiently large groups of people. We assume that the dynamics can be described by continuous functions of time, so that our mathematical models are represented by ordinary differential equations.

We mention here that any group of people, e.g. the 'population' of protesters or the larger community is not uniform but is structured according to various traits (Gavrilets, 2015). The choice of relevant traits depends on the context. For instance, in the context of social unrest the population of protesters can be structured according to the probability for a given individual to express a violent behaviour: indeed, although most of the Yellow Vest Movement participants protest peacefully, there is always a certain fraction of troublemakers that tend to behave aggressively. The larger community can be structured with regard to the probability for a given individual to join the protests; this, in turn, correlates with age, income, education, social background, etc. Whilst some people can be ignited easily (e.g. students), people from some other social groups might not so likely join the movement in any circumstances. Aiming to build tractable models and to avoid unnecessary complexity (cf. "Occam's razor"), in this paper we largely disregard the effect of individual differences. In particular, in Sections 2.1 and 2.2, we treat the protesters as a uniform group (but will introduce a population structure in Section 2.3). We also assume that the number of people in the society that could, under certain conditions, join the street actions is sufficiently large and hence is not a factor that limits the growth of the protesters number.

For the sake of simplicity, we neglect the spatial aspect of the protests. Nonspatial approach is justified if the focus is on a particular location where the protests take place. In case protests take place at several different locations across the country, we assume direct and indirect interactions between different groups cause the protesters behaviour to become uniform.

\subsection{Baseline 'single-species' model}

We describe the magnitude of the event by the single variable $N$ (measured in thousands) which is the total number of people actively participating in the street actions at a given time $t$ (measured in days), to which we will also refer as the 'population size'. In order to build a model that describes the dynamics of $N$ with time, we make the following assumptions:

1. The rate of change in the number of people attending the event is a result of the interplay between two processes, i.e. recruitment (people joining) and withdrawal (people quitting).

\footnotetext{
2 "Large scale random phenomena in their collective action create strict, non random regularity" (after Gnedenko \& Kolmogorov, (Gnedenko and Kolmogorov, 1954))
} 
2. Following earlier studies (Granovetter, 1978), we assume that recruitment is a collective phenomenon so that the recruitment rate is a nonlinear function of the number of people $N$ currently involved in the event. This assumption effectively means that the people who decide to join the protests are motivated mostly by social conformity (e.g. via influences of traditional news, social media, and their friends) and are not explicitly constrained by additional considerations such as costs and benefits.

3. Decision to withdraw from protests is made individually, so that the withdrawal rate is a linear function of the number of people participating in the event. The per capita withdrawal rate therefore does not depend on the number of protesters $N$. The per capita withdrawal rate monotonically increases with time as participants get tired or less motivated, or become busy with other issues requiring their time, attention and effort.

Under the above assumptions, we arrive at the following equation for the population size of the protesters:

$$
\frac{d N}{d t}=f(N) N-m(t) N
$$

where the first and second terms in the right-hand side are the recruitment rate and withdrawal rate, respectively, and $f(N)$ is the per capita recruitment rate. We mention here that Eq. (1) is a standard model of the population dynamics commonly used in ecology (Murray, 1989). We refer to it as the 'single-species model' meaning that there is only one dynamical variable, i.e. $N$.

To apply Eq. (1) to model the protests, we consider the per capita recruitment rate in the following form:

$$
f(N)=\epsilon+\frac{a N}{h^{2}+N^{2}}
$$

where $\epsilon, a$ and $h$ are positive parameters.

The per capita recruitment rate $f$ is therefore a non-monotonous function of $N$. It means that the rate at which new members are joining the movement tends to increase along with the number of people already in the movement for small $N$ but decreases for larger $N$. This is a typical behaviour response observed not only in humans but also in many animals; in particular, it is believed to be one of the mechanisms resulting in the Allee effect (Courchamp et al., 2008). When the number of people in the protests is sufficiently large, $N \gg h$, the per capita recruitment rate becomes approximately constant, $f(N) \approx \epsilon$. That reflects the fact that there is a certain fixed scale-independent probability of an average citizen to join protests, an assumption used in earlier studies (Turalska et al., 2013).

Consider first the case when per capita withdrawal rate $m$ is constant (see Fig. 1). Then there exist two critical values:

$$
m_{*}=\epsilon \quad \text { and } \quad m^{*}=\epsilon+\frac{\sqrt{2 a-1}}{2 h} .
$$

For any $m$, there is the trivial steady state $N=0$, it is stable for $m>m_{*}$ and unstable for $m<m_{*}$. For $m<m_{*}$ and $m>m^{*}, N=0$ is the only steady state of the system (cf. lines 1 


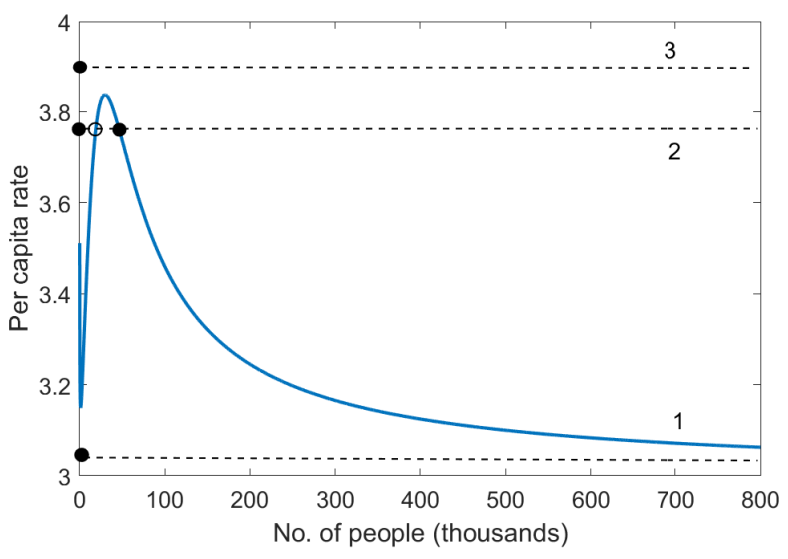

(a)

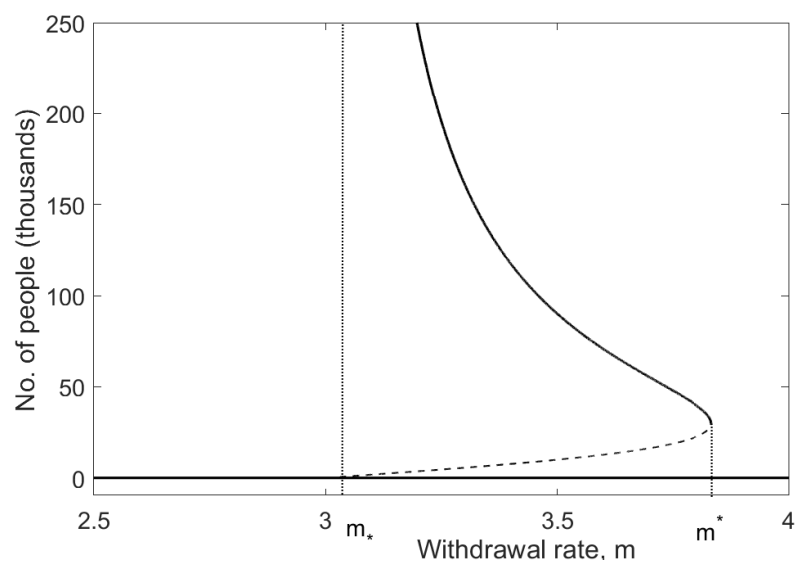

(b)

Figure 1: Properties of model (1-2) in the baseline case of constant $m$. (a) Blue curve shows $f(N)$, black dashed horizontal lines correspond to different values of $m$ (shown for three different cases). Steady states of the system defined by equation $f(N)=m$ arise as intersections between the blue and black lines. Large black dots show stable steady states, the open circle shows the unstable steady state. The system has two positive steady states in an intermediate range of $m$ (line 2 ) but only the trivial equilibrium for small (line 1) and large (line 3) values of $m$. (b) The bifurcation diagram: the steady state values of $N$ vs $m$.

and 3 in Fig. 1a). In the intermediate range $m_{*}<m<m^{*}$, the system possesses two positive steady states (see line 2), the upper one being stable and the lower one being unstable. The system experience a saddle-node bifurcation when $m$ passes $m^{*}$ and a transcritical bifurcation when $m$ passes $m_{*}$.

Next consider the case when the process is not stationary in the sense that the per capita withdrawal rate $m(t)$ explicitly depends on time. We assume that its initial value $m_{1}$ (i.e. at the beginning of unrest) is small. It then starts to increase monotonously eventually reaching a certain background value $m_{0}$. Note that, since $m$ has the dimension of inverse time, it can be written as $m(t)=1 / \tau(t)$ where $\tau$ can be interpreted as an average time of the individual participation in the protests. Correspondingly, the above assumption about the dependence of $m$ on time can be interpreted as follows: in the beginning, the people's expectations are high, the morale is high, and hence they are ready to participate in the protests for a long time. In the course of time, tiredness and disappointment may start growing hence reducing their willingness to stay engaged. The withdrawal rate $m$ in our model (1) is related to the social tension used in other studies (Berestycki et al., 2015) but plays the opposite role: it is small when the social tension in the society is high and it is large when the tension is low.

For computer simulations, we consider the following parametrization:

$$
m(t)=m_{1}+\left(m_{0}-m_{1}\right)\left(1-e^{-b t}\right),
$$

where $b$ is a parameter, so that $m(0)=m_{1}$ and $m(t)=m_{0}$ when $t \rightarrow \infty$.

We solved system (1-3) numerically. Since the protests are a result of social tension (sensu (Berestycki et al., 2015), see also Section 3), which is quantified in our model by $m$, we 


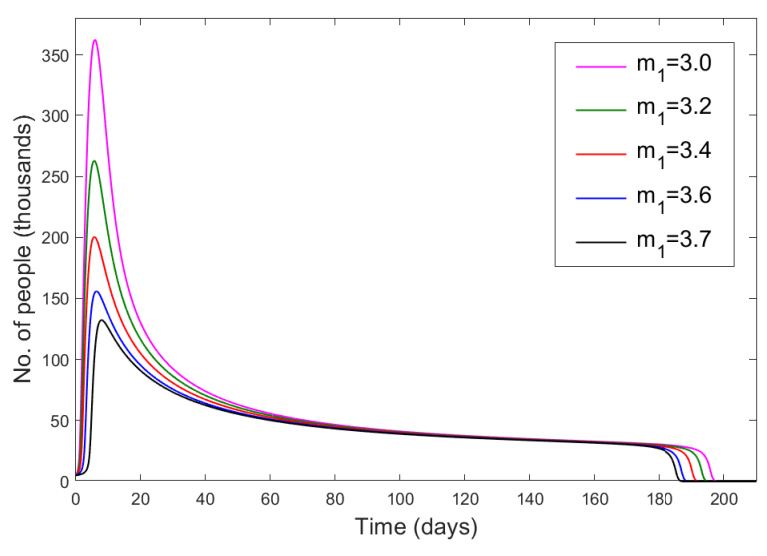

(a)

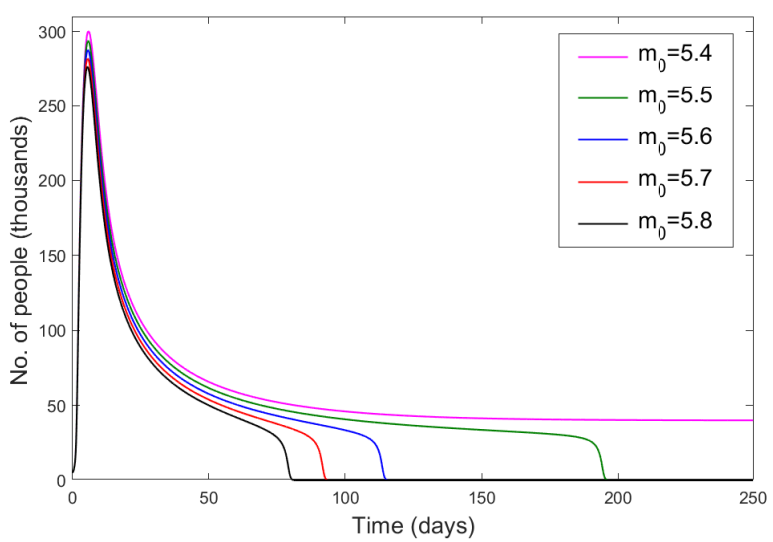

(b)

Figure 2: Solution of Eqs. (1-3) for different parameter values. (a) Effects of the initial value, $m_{1}$, with $m_{0}$ fixed at 5.124. (b) Effects of the final value, $m_{0}$, with $m_{1}$ fixed at 3.0. Other parameters are given in the text.

consider $m_{0}$ and $m_{1}$ as the controlling parameters. The solutions obtained for different values of $m_{0}$ and $m_{1}$ are shown in Fig. 2 (for other parameters fixed at some hypothetical values as $\epsilon=3, a=146.47, h=34.54$ and $b=0.01)$. It is readily seen that, in a certain range of values of $m_{0}$ and $m_{1}$, the solution possesses properties consistent with the dynamics of the protests: in all cases shown in Fig. 2, the dependence of the population size on time exhibits a peak followed by a gradual decay. Interestingly, the slow decay is eventually followed by a fast decay to zero. This is the effect of the ghost attractor (Hastings et al., 2018, Morozov et al., 2019, Strogatz, 1994). When an increase in $m$ brings the system past the saddle-node bifurcation, the system does not possess a positive steady state any more. However, in case the 'final' value $m_{0}$ is only slightly larger than the critical value $m^{*}$, the rate of change for the values of $N$ close to its pre-bifurcation steady state value remains very slow. This slow change accounts for the 'shelf' in the solution shape. Eventually, the system leaves the area of the phase space with the slow dynamics and then experiences a fast transition towards the origin, which, for $m_{0}>m^{*}$, is the global attractor of the system. Note that if $m_{0}<m^{*}$, the positive attractor exists and the population size never reaches zero but instead stabilizes at some positive value; see the magenta curve in Fig. $2 \mathrm{~b}$.

Being encouraged by the apparent qualitative agreement between the solution properties and the generic features of social protests, now we are going to consider whether model (1-3) can provide a good description of the data on the Yellow Vest Movement. The number of protesters attending the weekly street actions (normally on Saturdays) is shown in Fig. 3. We first notice that its dependence on time after the main peak during the first week of protests is not monotonous; it first shows a decrease to a relatively small value during the seventh and eighth weeks of the protests then increasing to a secondary peak before starting showing a slow gradual decay. Here we hypothesize that the decrease to the local temporal minimum is the effect of the calendar rather than a feature of the intrinsic dynamics of the movement, and there is considerable amount of anecdotal evidence of that in the French mass media 
(J.C. Poggialle, private communication). Indeed, it is readily seen that the dates for the local minimum fall into the Christmas/New Year season when for many potential protesters their participation in the street actions could be hindered by their family duties. We therefore do not attempt to describe the local minimum and secondary maximum but instead focus on the main peak and the slowly decreasing tail of the graph.

In order to fit the model (1-3) to the data, we performed the gradient search in the parameter space aiming to minimize RSS (residual sum-of-squares). The best-fitting solution is obtained for $a=152.3287, h=30.6475, m_{1}=3.126, b=0.031, \epsilon=3, m_{0}=5.5$ and $N(0)=5$ and is shown in Fig. 3 by the blue curve. Apparently, it is in a good agreement with the existing data up to date. We mention here that, although the above parameter set provides the best fit, a reasonably small variation of parameter around their best-fitting values do not change the properties of the solution much; this is readily seen in Fig. 2. In particular, the long transient slow decay is clearly seen for a range of values of $m_{1}$ and $m_{0}$.

Interestingly, the model predicts that the movement will soon come to an abrupt end without any action from the French government, simply due to its inherent dynamical features, because the slow decay of the solution is in fact transient dynamics caused by the ghost attractor.

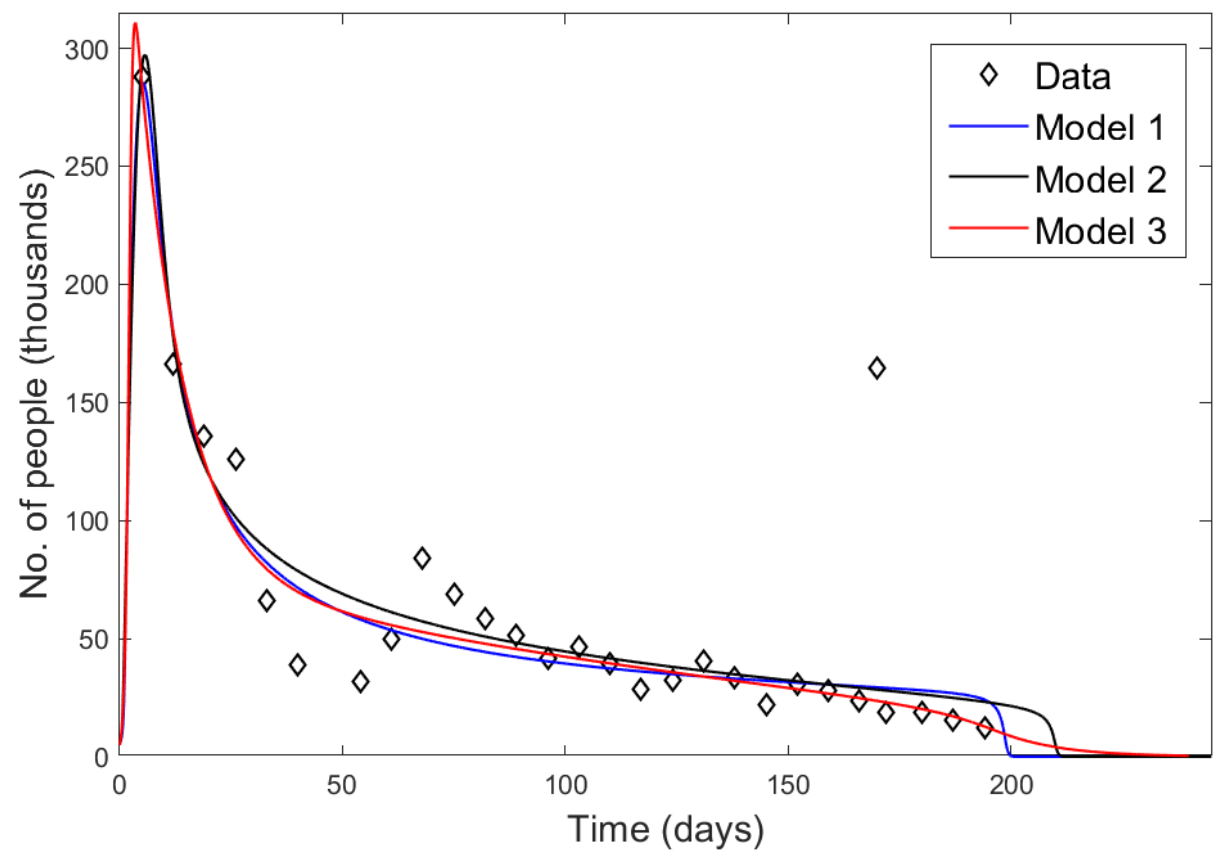

Figure 3: Number of the Yellow Vest Movement protesters vs time described by different models: diamonds show the data, blue curve for Model 1 (Eqs. (1-3)), black curve for Model 2 (Eqs. (4) with (2) and (5)), red curve for Model 3 (Eqs. (6-9)). Parameters are given in the text. The data are taken from (Wikipedia, 2019). The models are fitted to the data by minimizing RSS. The standalone point in the middle of the figure corresponds to the street actions on May 1; in our analysis it was regarded as outlier and was not taken into account in the best-fitting procedure. 


\subsection{Two-species models}

In the previous section, the per capita withdrawal rate was assumed to be a given function of time. Although this approach has its rationale (see the paragraph preceding Eq. (3)), it should be considered as a special case because it disregards potential feedbacks, e.g., possible dependence of $m$ on the number of protesters $N$. Meanwhile, there is a variety of reasons to expect such dependence. For instance, the number of protesters is likely to have an effect on the level of social tension in the society, especially when $N$ is sufficiently large. Indeed, the larger the number of people involved in protests is, the more likely it is that (partial) goals of protests are achieved (e.g. to change the government policy). Once this happens, the social tension decreases; this makes the protests less justified and hence the protesters' withdrawal rate is likely to increase. There can be other mechanisms too, one of them will be discussed below.

A more general modelling approach to describe the dynamics of protests should therefore consider $m$ as a dynamical variable. In order to build a model, we keep assumptions 1 and 2 from the previous section but change assumption 3 so that the rate of change in $m$ can depend on $N$. As an immediate yet nontrivial extension of model (1-3), we then arrive at the following system:

$$
\frac{d N}{d t}=f(N) N-m N, \quad \frac{d m}{d t}=G(m, N),
$$

where $G$ is a certain function to be specified, its form depending on the feedback mechanism.

Apparently, model (4) is more general than model (1-3) and hence can describe a broader variety of situations. For a particular choice of function $G=b\left(m_{0}-m\right)$, it includes model $(1-3)$ as a special case.

There can be various lines of reasoning to determine function $G$. Here we take into account that protests usually bring economic damage. It includes direct damage such as broken shop windows, burnt cars, etc. and also indirect damage, e.g. through the disruption of public transport. We assume that the amount of damage caused is proportionate to the number of people involved in the street actions. We then further assume that an ordinary protest participant eventually develops the feeling of guilt for causing damage to the community, and that is likely to increase the chances for him to withdraw from the street actions. Correspondingly, it means that the rate of change in $m$ should be an increasing function of the damage, which we consider to be linear, and hence proportionate to the number of protesters. We therefore arrive at the following equation for the withdrawal rate:

$$
\frac{d m}{d t}=\beta N
$$

where $\beta$ is a coefficient.

The population dynamics of protests is then described by Eqs. (4) with (2) and (5). We refer to these equations as Model 2. Since the damage caused by the protests is the essential element of the model, we consider $\beta$ as the controlling parameter. Having in mind that the Yellow Vests protests have been largely peaceful, we consider that the average damage per protester per unit time is small; hence relevant values of $\beta$ should be small too. 


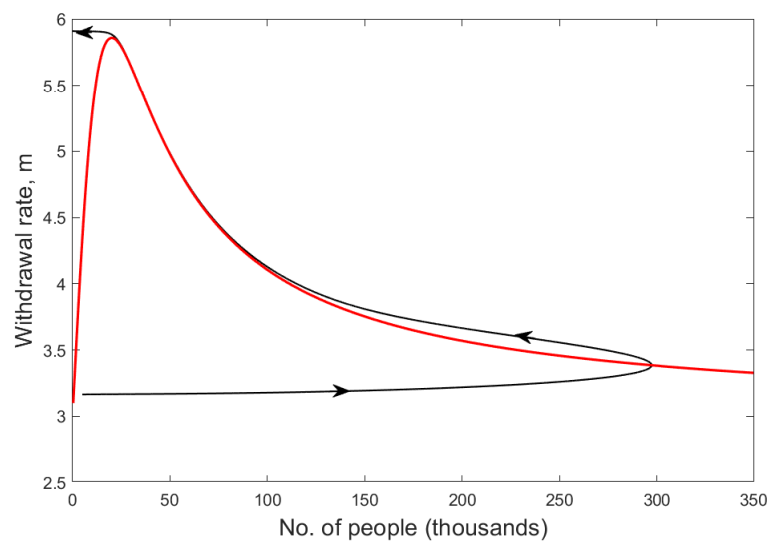

(a)

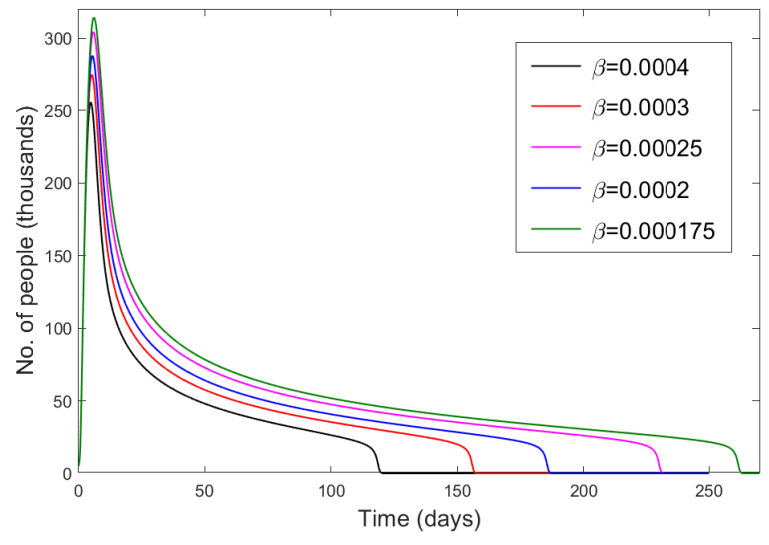

(b)

Figure 4: (a) Phase plane of system (4-5) (with $f(N)$ given by Eq.(2)); blue curve is the isocline $\frac{d N}{d t}=0$, black curve shows the system trajectory corresponding to a solution exhibiting long transient dynamics. (b) Response of the solution shape to the variation in parameter $\beta$.

Figure 4 a shows the phase plane of system (4-5). We readily notice that the system does not possess a positive steady state and hence a ghost attractor cannot emerge. In spite of that, the solutions of the system clearly exhibit long transient dynamics. The main peak is followed by a long period of slow decay in the protesters number, the smaller parameter $\beta$ the longer the duration of the transient stage (see Figure $4 \mathrm{~b}$ obtained for parameters $\epsilon=3, a=$ 114.991, $h=20.116$, the initial conditions $N(0)=5, m(0)=3.1646$, and different values of $\beta$ ). This is due to a different mechanism; long transients in system (4-5) are a consequence of the slow-fast dynamics (Hastings et al., 2018, Kooi and Poggiale, 2018, Morozov et al., 2019).

Figure 3 shows how the prediction of Model 2 (black curve) agrees with the data on the Yellow Vests protests, the best-fitting parameter values $(\epsilon=3, a=114.9908, h=20.1161$ and $\beta=0.00022$, with the initial conditions used as $N(0)=5$ and $m(0)=3.1646)$ being found by minimizing RSS. Similarly to Model 1, Model 2 too predicts an abrupt end of the protests within the next few weeks.

\subsection{Multi-species model: protests 'epidemics'}

In many previous studies, the spread of rebellious behaviour and revolutionary ideas was considered as a certain 'epidemics' (Epstein, 2002). Indeed, the underlying assumptions appear to be quite similar between the diseases dynamics and for the spread of ideas, behaviors or habits (van Voorn and Kooi, 2013). In either case, there must be a direct interaction, i.e. a contact between a person who is 'infected' with certain ideas (or behaviour) and a 'susceptible' person who is not yet infected but can, with a certain probability, accept and utilize the new ideas or adopt new behaviour. The dynamical properties of the disease spread and those of the spread of behaviours show considerable similarity too, in particular predicting quasi-

periodical outbreaks of the 'disease' (Epstein, 2002). Correspondingly, in this section we 


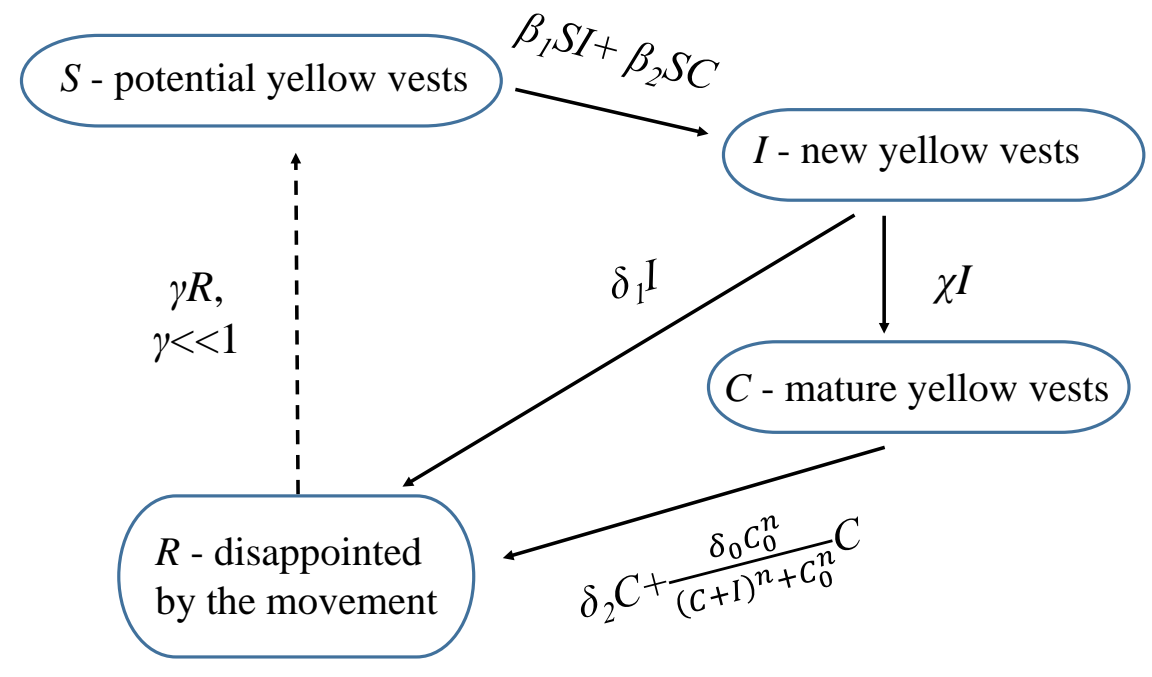

Figure 5: The structure of the epidemic-type model of the Yellow Vests protests.

endeavor to model the dynamics of protests using an epidemiological approach and to apply it to the Yellow Vest Movement.

More specifically, we adapt the ideas behind the SIR-type model (Martcheva, 2016). The system consists of four compartments shown in Fig. 5. Here $S$ are potential protest participants, $I$ are the new protesters who joined the movement recently, $C$ are experienced 'mature' protesters and $R$ are the former protesters how became disappointed or disillusioned and withdrew from the movement. Correspondingly, the model consists of the following equations:

$$
\begin{aligned}
\frac{d S}{d t} & =-\beta_{1} S I-\beta_{2} S C+\gamma R \\
\frac{d I}{d t} & =\beta_{1} S I+\beta_{2} S C-\chi I-\delta_{1} I \\
\frac{d C}{d t} & =\chi I-C\left(\delta_{2}+\delta_{0} \frac{C_{0}^{n}}{(C+I)^{n}+C_{0}^{n}}\right), \\
\frac{d R}{d t} & =\delta_{1} I+C\left(\delta_{2}+\delta_{0} \frac{C_{0}^{n}}{(C+I)^{n}+C_{0}^{n}}\right)-\gamma R,
\end{aligned}
$$

where the parameters have their usual epidemiological meaning.

Note that the transition from $C$ to $R$ (i.e. from the class of experienced protesters to the disappointed cohort) is described by two terms. We therefore assume that the rate at which the experienced protesters become disappointed and withdraw from the movement is sizedependent in a manner similar to the Allee effect (Courchamp et al., 2008): it reaches its largest value $\left(\delta_{0}+\delta_{2}\right)$ when $C$ is small and decreases monotonously to its minimum value $\left(\delta_{2}\right)$ when $C$ is large. This transition is smooth for $n \sim 1$ but becomes threshold-like for $n>1$. We also assume that the withdrawal rate for the experienced protesters $(C)$ is substantially smaller than that for those who recently joined the movement $(I)$, so that $\delta_{2} \ll \delta_{1}$. We also consider the recovery rate from $R$ to $C$ (quantified by $\gamma$ ) to be very low, ultimately zero. 


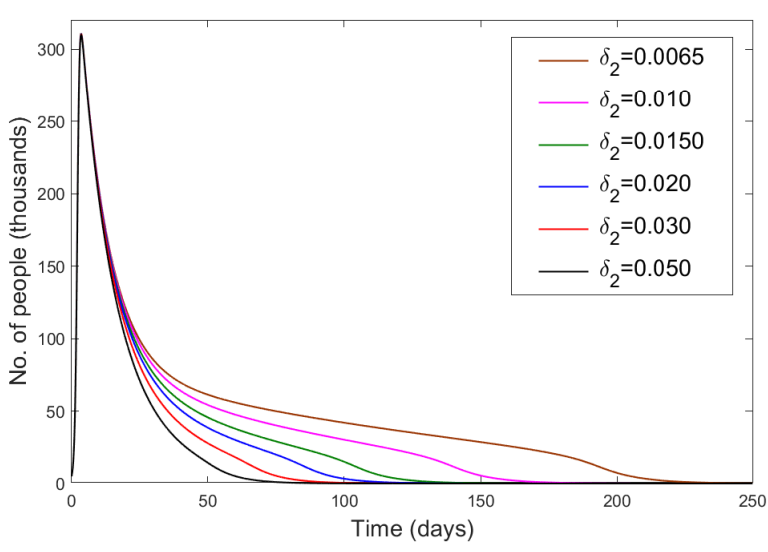

(a)

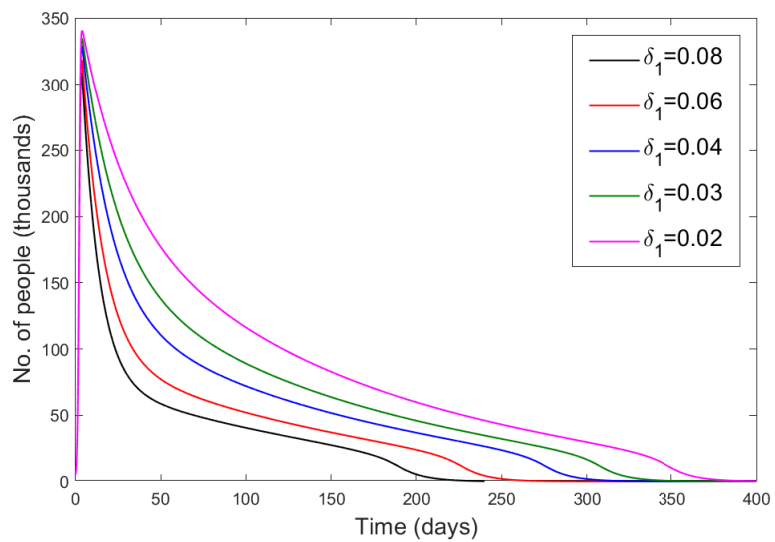

(b)

Figure 6: Number of protesters $(I+C)$ vs time obtained from Eqs. (6-9) for different parameter values. (a) Response of the solution shape to changes in $\delta_{2}$ for $\delta_{1}=0.0762$, other parameters and the initial conditions are given in the text. (b) Response of the solution shape to changes in $\delta_{1}$ for $\delta_{2}=0.0069$, other parameters are the same as above.

System (6-9) is solved numerically; typical solutions obtained for different parameter values are shown in Fig. $6\left(\beta_{1}=0.0045, \beta_{2}=0.1832, \chi=0.0203, \gamma=0, n=5, C_{0}=15\right.$ and $\delta_{0}=\delta_{1}-\delta_{2}$ with the initial conditions $S(0)=350, I(0)=5, C(0)=0$ and $\left.R(0)=0\right)$. The model once again exhibits long transient dynamics which become more prominent for smaller values of $\delta_{2}$. The overall number of protesters $(I+C)$ agrees well with the Yellow Vests

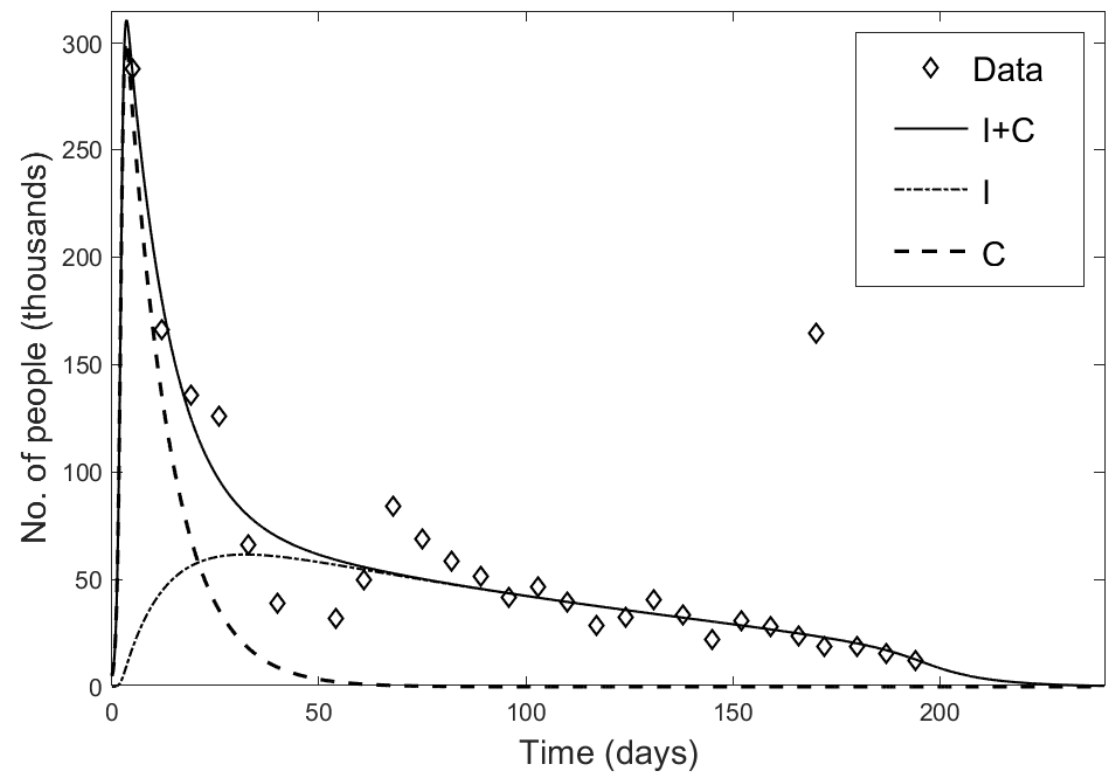

Figure 7: Structured dynamics of the Yellow Vest Movement as predicted by model (6-9): the dashed curve shows the number of new protesters and the dotted curve shows the number of experienced 'mature' protesters. Parameters are the same as in Fig. 3. 
data; see the best-fitting red curve in Fig. 3 obtained by minimizing RSS (the corresponding parameters are $\delta_{1}=0.0762$ and $\delta_{2}=0.0064$, other parameters the same as above). Similarly to the models from the previous sections, model (6-9) predicts a rather abrupt end of the movement in just several weeks, i.e. by mid-July 2019 at latest.

Note that, since model (6-9) contains more details about the protests dynamics, it allows us to make more specific prediction too. In particular, it allows one to consider the dynamics of new and experienced movement participants separately; see Fig. 7. One can see that the existence of the long transient - the long tail in the model's solution - is explained by the growth and persistence of experienced protesters. This appears to be in a good agreement with reports in the mass media that the core group of the movement was created shortly after the start of demonstrations where individuals with similar beliefs, ideas and social demands had met and established a strong network (Huret, 2019).

\section{Comparison with the Berestycki et al. model}

In this section, we will further strengthen our argument about the importance of long transients in the dynamics of the Yellow Vests protests by analyzing an earlier model of social unrest developed in (Berestycki et al., 2015). We will show that its dynamical behavior are qualitatively similar to those of our models described above.

Berestycki et al. (2015) described the dynamics of protests with two dynamical variables, namely, the level of protests activity $N$, which is roughly equivalent to the total number of protesters involved in the street actions, and the "social tension" (say, $\alpha$ ). The latter is a proxy of the quality of life and social justice more generally and hence quantifies the level of resentment that the society feels toward the relevant authorities. They assumed that the protesters recruitment rate is a monotonously increasing function of the social tension. Neglecting the spatial structure, their model can be written as follows:

$$
\frac{d N}{d t}=-\omega N+r(\alpha) F(N), \quad \frac{d \alpha}{d t}=-p(N) \alpha+\alpha_{0} .
$$

We consider the same functional forms for $r(\alpha)$ and $p(N)$ as in Berestycki et al. (2015); for the growth rate $F(N)$, in order to make model (10) comparable with our models developed in Sections 2.1 and 2.2, we parametrize it as in Eq. (2) (instead of the logitic growth considered in (Berestycki et al., 2015)). Equations (10) then take the following form:

$$
\begin{aligned}
\frac{d N}{d t} & =-\omega N+\frac{N}{1+\exp (-\beta \alpha)}\left(\epsilon+\frac{a N}{h^{2}+N^{2}}\right) \\
\frac{d \alpha}{d t} & =-\frac{\theta \alpha}{\left(N_{0}+N\right)^{k}}+\alpha_{0},
\end{aligned}
$$

where $\omega, \alpha_{0}, \beta, \theta, N_{0}$ and $k$ are parameters of the model.

Analysis of Eqs. (11-12) readily reveals that, for certain parameter values (e.g. as a response to a decrease in $\alpha_{0}$ or $N_{0}$ ), the corresponding dynamical system undergoes a saddle-node bifurcation (see Fig. 8); as a result, a ghost attractor is born. For parameter values in the 


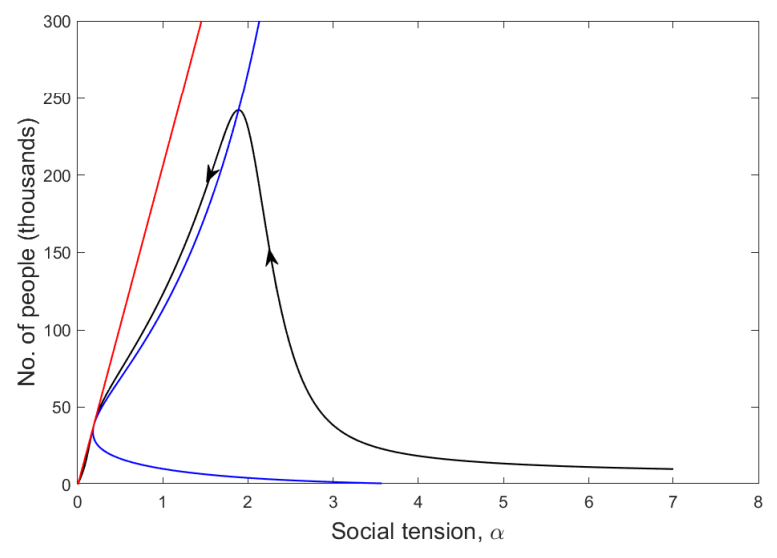

(a)

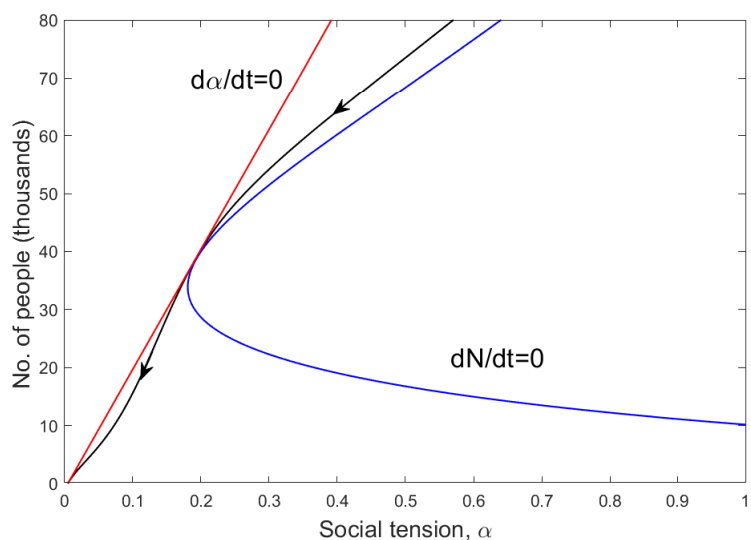

(b)

Figure 8: Phase plane of the Berestycki et al. model (11-12). (a) The relative position of the system's isoclines (blue curve for Eq.(11), red line for Eq.(12)) for a value of the parameters just past the saddle-node bifurcation. As a result of the bifurcation, a ghost attractor has emerged. The black curve shows a system trajectory passing through the location of the ghost attractor. (b) A magnified view of panel (a).

supercritical range (but not too far away from their critical values), the dynamics of the system are dominated by the ghost attractor resulting in long transients. A relevant solution of model (11-12) is shown in Fig. 9 (the corresponding system's trajectory is shown in Fig. 8 by the black curve) obtained for parameters $\epsilon=3, a=160, \alpha_{0}=0.1, \omega=2.8426, \theta=20.6901, \beta=$ $0.6679, h=33.87$ and the initial conditions $\alpha(0)=7.0024$ and $N(0)=10$. It has properties typical for the long transient dynamics: the peak in the number of protesters is followed by

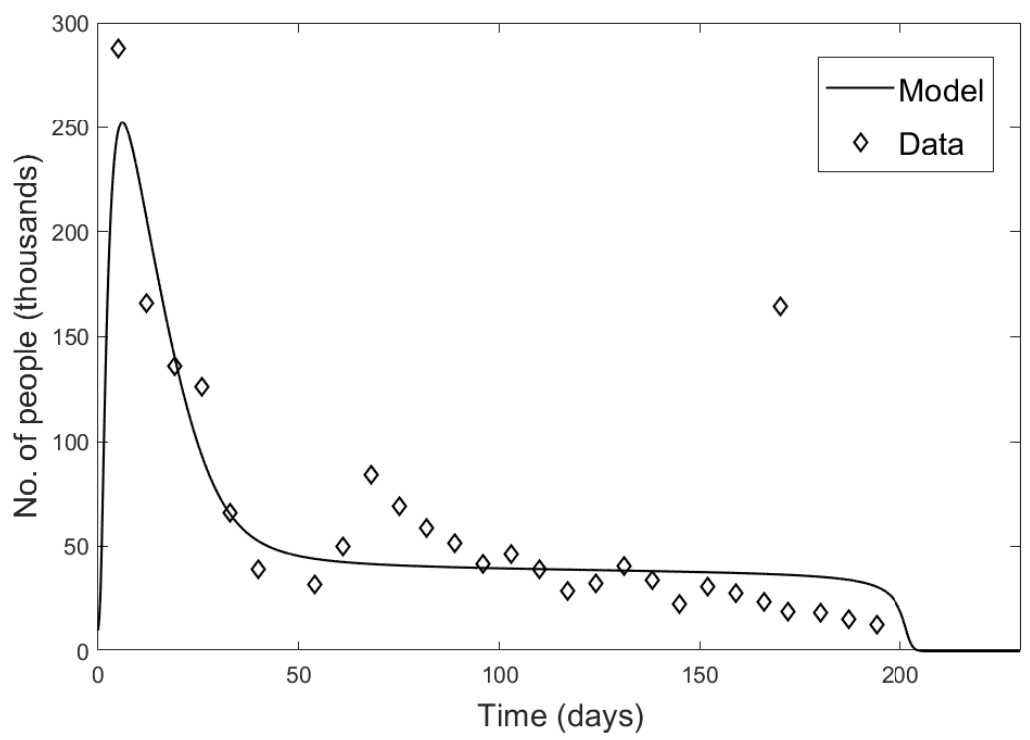

Figure 9: Comparison of the Berestycki et al. model (11-12) with data on Yellow Vest Movement. 
a long period of time when the number changes very slowly. The slow change is eventually followed (when the system leaves the location of the ghost attractor) by a fast drop to zero.

A natural question arises here as to which model provides a better description of the data on the Yellow Vests protests, i.e. Models 1, 2 or 3, or model (11-12) to which we refer as Model 4. We performed a thorough statistical analysis of the quality of the data fit, in particular taking into account that different models have different number of parameters. The results are shown in Table 1. We therefore conclude that Model 3 gives considerably better description of the data than any of the other models. Interestingly, it appears that the Berestycki at al. model provides slightly worse agreement with the data on the Yellow Vest Movement than our models developed in the previous section.

Table 1: Statistical measures defining the quality of data fitting with different models. Here RSS stands for the residual sum-of-squares and $\Delta w$ for the Akaike weight.

\begin{tabular}{|l|c|c|c|c|c|}
\hline & RSS & $R^{2}$ & AIC & $\Delta$ AIC & $\Delta w$ \\
\hline \hline Model 1 (Section 2.1) & 5702 & 0.4312 & 241.5 & -2.25 & 0.175 \\
Model 2 (Section 2.2) & 5495 & 0.45 & 241.5 & -1.25 & 0.287 \\
Model 3 (Section 2.3) & 4871 & 0.514 & 239.5 & 0 & 0.538 \\
Model 4 (Section 3) & 9915 & 0.11 & 258.44 & -19.19 & $3.66 \cdot 10^{-5}$ \\
\hline
\end{tabular}

\section{Discussion}

In this study, we highlight the role of long transients in the dynamics of social protests. To the best of our knowledge, the effect of long transients on social dynamics has been largely overlooked in the literature. A fingerprint of this phenomenon is the presence of a long period of time (slow phase) when the number of protesters changes only slightly, which is eventually followed by a fast decay in their number (fast phase) and the disappearance of the protests. Our study is motivated by the ongoing Yellow Vest Movement in France. It started in November 2018 and has been going on for six months already and is now regarded as exceptionally long by the mass media (Meyers-Belkin, 2019). Understanding the effect of long transients in social protests and civil disobedience is a problem of high practical importance, in particular for the authorities to efficiently control the situation and prevent the collapse of the system. The policy-makers need to take into account the possibility that, under some particular conditions, the duration of the unrest can be very long simply due to their inherent properties. In the modern history, there were similar situations where violent social protests persisted over months or even years (hence indicating long-term transient dynamics). One well-known example is the Russian strikes movement in 1905-1907 (Haynes, 2005, Perrie, 1972) where a large number of protests in 1905 was then followed by a two-year long 'tail' of actions of a smaller magnitude.

On the other hand, long transients in the dynamics of social unrest are not observed for all protests: often there is a fast decay in the number of protesters with time, e.g. in case 
of the London riots in 2011 (Davies et al., 2012) and French riots in 2005 (Bonnasse-Gahot et al., 2018). Our models allow to distinguish between the patterns with fast and slow decay at the tail in terms of the model parameters. In particular, as we showed in Section 2.1, for the long transient dynamics to occur in Model 1 parameter $m_{0}$ should be sufficiently close to its bifurcation value; see Figs. 1 and 2. For long transients to occur in Model 2 (see Section 2.2 ), parameter $\beta$ should be sufficiently small.

Our models reveal the mechanisms of long transients in the dynamics of social unrest: this is due to the ghost attractors and slow-fast dynamics, which have been previously reported in the general theory of dynamical systems (Hastings et al., 2018, Lai and Tél, 2011, Strogatz, 1994). Note that the long transients due to the above mechanisms can occur in a variety of models of social dynamics including models well known from the literature (Berestycki et al., 2015); see Section 3. This leads to a conclusion that the long transients in social dynamics are likely to be ubiquitous.

Application of our models to the data on the Yellow Vest Movement leads to the prediction that they will seize abruptly, although the specific date of the end of the movement varies between the models ranging from mid-June 2019 (Model 1) to mid-August 2019 (Model 3). Note that the end of the movement is predicted to occur simply due to the inherent properties of the corresponding social dynamics. Thus, the best strategy of the French authorities might be to avoid any direct action to allow the movement to get exhausted by itself. However, our modelling approach also shows that the potential problem with this passive strategy is that a relatively small external perturbation (shock) might trigger a new large amplitude peak of protests to restart the overall cycle again.

Note that the proportion of arrested people the London riots in 2011 (estimated as 25-30\%, (Davies et al., 2012) also (Wikipedia, 2011) and further references therein) and in the French riots in 2005 (estimated as 12\%, see (Bonnasse-Gahot et al., 2018) and references therein) was much higher than in the case of the Yellow Vests protests (just about 3\% of the maximal number of protesters on November 17th, 2018 (Wikipedia, 2019)). This can be explained by the fact that the latter should be considered as a social movement rather than a riot even that acts of violence were reported during the protests, especially in first few months. From the modelling point of view, this means that the level of social tension for political protests may decrease at slower rate than in the case of a typical riot. On the other hand, the motivation to stay in the social movement decreases slower than in riots. In Models 1 and 2 this means a slow increase of the quantity $m(t)$ which leads to a long term transient via a ghost attractor; see Figs. 1-2.

In conclusion, we acknowledge that a number of other factors and processes neglected in our approach can play a crucial role in the dynamics of protests, for example, the heterogeneity of the population in terms of beliefs, material and normative benefits and costs, susceptibility to news, propaganda, and counter-propaganda, social status, social networks, etc. (Gavrilets, 2015, Homer-Dixon and et al., 2013, Mistry et al., 2015, Raafat et al., 2009). Heterogeneity of space can be also an important factor affecting the dynamics of protests as it was shown in a number of studies (Berestycki et al., 2015, Bonnasse-Gahot et al., 2018, Davies et al., 2012). The recruitment of protesters can be significantly affected by activists, as it happened in the 
case in the Russian revolutions in 1905-1907 and later in 1917 (Andreev et al., 1997, Haynes, 2005, Perrie, 1972). Nowadays such activists are active internet users and social engineers and hence may have even a stronger influence. However, from the general perspective of the dynamical systems theory, we expect more realistic models of social protests to exhibit long-transient dynamics as well.

\section{Acknowledgements}

This work was supported by the NIMBioS through the Working Group "Long-term Transient Dynamics and Ecological Forecasting". SG was supported by the U. S. Army Research Office grants W911NF-14-1-0637 and W911NF-17-1-0150, the Office of Naval Research grant W911NF-18-1-0138, the National Institute for Mathematical and Biological Synthesis through NSF Award \#EF-0830858, and by the University of Tennessee, Knoxville. AM and SP are thankful to JC Poggiale for illuminating and helpful discussions of the problem.

\section{References}

Andreev, A., Borodkin, L., and Levandovskii, M. (1997). Using methods of non-linear dynamics in historical social research: application of chaos theory in the analysis of the worker's movement in pre-revolutionary russia. Historical Social Research, 22(3/4), 64-83.

BBC News, U. (2013). 2011 england riots. https://www.bbc.com/news/world-europe21004322.

Berestycki, H. and Rodriguez, N. (2016). Analysis of a heterogeneous model for riot dynamics: the effect of censorship of information. Euro. Jnl of Applied Mathematics, 27, 554582.

Berestycki, H., Nadal, J.-P., and Rodguez, N. (2015). A model of riots dynamics: shocks, diffusion and thresholds. Networks and Heterogeneous Media, 10, 443-475.

Bonnasse-Gahot, L., Berestycki, H., and Depuiset, M. (2018). Epidemiological modelling of the 2005 french riots: a spreading wave and the role of contagion. Sci. Rep., 8, 107.

Brantingham, P. and et al. (2012). The ecology of gang territorial boundaries. Criminology, 50, 851-885.

Centola, D. M. (2013). Homophily, networks, and critical mass. Rationality and Society, 25, $3-40$.

Courchamp, F., Berek, L., and Gascoigne, J. (2008). Allee Effects in Ecology and Conservation. Oxford University Press, Oxford.

Davies, T., Fry, H., A.G., W., and Bishop, S. (2012). A mathematical model of the london riots and their policing. Sci. Rep., 3, 1303. 
Eguiluz, V., Zimmermann, M., Cela-Conde, C., and San Miguel, M. (2006). Cooperation and emergence of role differentiation in the dynamics of social networks. arXiv:physics/0602053v1.

Epstein, J. (1997). Nonlinear Dynamics, Mathematical Biology, and Social Science. AddisonWesley, Reading MA.

Epstein, J. (2002). Modeling civil violence: An agent-based computational approach. PNAS, 99(suppl. 3), 7243-7250.

Fonoberova, M. and et al. (2012). Nonlinear dynamics of crime and violence in urban settings. Journal of Artificial Societies and Social Simulation, 15(1), 2.

Gavrilets, S. (2015). Collective action problem in heterogeneous groups. Phil. Trans. R. Soc. Lond. B, 370, 20150016.

Gavrilets, S. and Hastings, A. (1995). Intermittency and transient chaos from simple frequency-dependent selection. Proceedings of the Royal Society London B, 261(1361), 233238.

Gavrilets, S. and Hastings, A. (1998). Coevolutionary chase in two-species systems with applications to mimicry. Journal of Theoretical Biology, 191(4), 415-427.

Gnedenko, B. and Kolmogorov, A. (1954). Limit Distributions for Sums of Independent Random Variables. Addison-Wesley, Cambridge MA.

Granovetter, M. (1978). Threshold models of collective behavior. American Journal of Sociology, 83, 1420-1443.

Hastings, A. and Higgins, K. (1994). Persistence of transients in spatially structured ecological models. Science, 263, 1133-1136.

Hastings, A., Abbott, K., Cuddington, K., Francis, T., Gellner, G., Lai, Y., Morozov, A., Petrovskii, S., Scranton, K., and Zeeman, M. (2018). Transient phenomena in ecology. Science, 361, eaat6412.

Haynes, M. (2005). Patterns of conflict in the 1905 revolution. In P. Glatter, editor, The Russian Revolution of 1905: Change Through Struggle, page 215233. Porcupine Press, London.

Homer-Dixon, T. and et al. (2013). A complex systems approach to the study of ideology: cognitive-affective structures and the dynamics of belief systems. J. Soc. Polit. Psychol., 1(1), 337-363.

Huret, R. (March 25th 2019). Les célibataires au coeur du grand débat? Libération. fr.

Jakimowicz, A. (2010). Catastrophes and chaos in business cycle theory. Acta Phisica Polonica A, 117(4), 640-646. 
Jakimowicz, A. (2013). Characteristics of Complexity in Selected Economic Models in the Light of Nonlinear Dynamics. Acta Phisica Polonica A, 123(3), 542-546.

Khosaeva, Z. (2015). The mathematics model of protests. Comp. Res. Modeling, 7(6), 13311341.

Kooi, B. and Poggiale, J. (2018). Modelling, singular perturbation and bifurcation analyses of bitrophic food chains. Mathematical Biosciences, 301, 93-110.

Lai, Y. and Tél, T. (2011). Transient Chaos - Complex Dynamics on Finite-Time Scales. Springer, New York.

Macy, M. W. (1991). Chains of cooperation: threshold effects in collective action. American Sociological Review, 56, 730-747.

Martcheva, M. (2016). An Introduction to Mathematical Epidemiology. Springer, New York.

Meyers-Belkin, H. (April 26th 2019). Macron to announce measures to allay yellow vest rage. France 24.

Mistry, D., Zhang, Q., Perra, N., and Baronchelli, A. (2015). Committed activists and the reshaping of the status-quo social consensus. Phys. Rev. E, 92, 042805.

Morozov, A., Abbott, K., Cuddington, K., Francis, T., Gellner, G., Hastings, A., Lai, Y., Petrovskii, S., Scranton, K., and Zeeman, M. (2019). Long transients in ecology: theory and applications. Physics of Life Reviews, page submitted.

Murray, J. (1989). Mathematical Biology. Springer, Berlin.

Perrie, M. (1972). The russian peasant movement of 1905-1907: Its social composition and revolutionary significance. Past \& Present, 57, 123-155.

Petrovskii, S., Morozov, A., and Li, B.-L. (2005). Regimes of biological invasion in a predatorprey system with the allee effect. Bulletin of Mathematical Biology, 67, 637-661.

Pires, B. and Crooks, A. (2017). Modeling the emergence of riots: A geosimulation approach. Computers, Environment and Urban Systems, 61, 66-80.

Raafat, R., Chater, N., and Frith, C. (2009). Herding in humans. Trends in Cognitive Sciences, 13(10), 420-428.

Schussman, A. and Soule, S. (2005). Process and protest: Accounting for individual protest participation. Social Forces, 84(2), 1083-1108.

Smith, L., Bertozzi, A., Brantingham, P., Tita, G., and Valasik, M. (2012). Adaptation of an ecological territorial model to street gang spatial patterns in los angeles. Discrete and Continuous Dynamical Systems, 32(9), 3223-3244. 
Strogatz, S. (1994). Nonlinear Dynamics and Chaos. Perseus Books Publishing, Cambridge MA.

Turalska, M., West, B., and Grigolini, P. (2013). Role of committed minorities in times of crisis. Sci. Rep., 3, 1371.

Turchin, P. (2003). Historical Dynamics: Why States Rise and Fall. Princeton University Press, Princeton, NJ.

Turchin, P., Gavrilets, S., and Goldstone, J. (2018). Linking micro to macro models of state breakdown to improve methods for political forecasting. Cliodynamics: The Journal of Quantitative History and Cultural Evolution, 8, 159-181.

Van Geest, G., Coops, H., Scheffer, M., and van Nes, E. (2005). Long transients near the ghost of a stable state in eutrophic shallow lakes with fluctuating water levels. Ecosystems, 10, 36-46.

van Voorn, G. and Kooi, B. (2013). Smoking epidemic eradication in a eco-epidemiological dynamical model. Ecological Complexity, 14, 180-189.

Whitehouse, H., Jong, J., Buhrmester, M., Gomez, A., Bastian, B., Kavanagh, C., Newson, M., Matthews, M., Lanman, J., McKay, R., and Gavrilets, S. (2017). The evolution of extreme cooperation via shared dysphoric experiences. Sci. Rep., 7, 44292.

Wikipedia (2011). 2011 England riots. https://en.wikipedia.org/wiki/2011-England-riots.

Wikipedia (2019). Gelbwestenbewegung. https://de.wikipedia.org/wiki/Gelbwestenbewegung. 